\title{
Study on Quantum Annealing Using the Density Matrix Renormalization Group
}

\author{
Sei SUZUKI ${ }^{1 *}$ and Masato OKADA ${ }^{2,3 \dagger}$ \\ ${ }^{1}$ Department of Physics, Tokyo Institute of Technology, Oh-okayama, Meguro 152-8551, Japan \\ ${ }^{2}$ Graduate School of Frontier Sciences, The University of Tokyo, Kashiwa 277-8561, Japan \\ ${ }^{3}$ Brain Science Institute, RIKEN, Wako 351-0198, Japan
}

Received October 31, 2006; final version accepted December 28, 2006

\begin{abstract}
Quantum annealing is a quantum algorithm proposed recently for combinatorial optimization problems. It manipulates time evolution of a quantum mechanical state and obtain an approximate solution. In order to implement the algorithm in classical computers, we propose to apply the density matrix renormalization group method. Simulation of the time evolution of a quantum mechanical state becomes possible by the density matrix renormalization group method for problems of large size. We explain quantum annealing and the density matrix renormalization group method, and present results of numerical simulation using them.
\end{abstract}

KEYWORDS: quantum annealing, density matrix renormalization group

\section{Introduction}

Combinatorial optimization is probably the most challenging task in computer science. In various kinds of combinatorial optimization problems, there exist essentially hard problems. In such problems, the number of combination increases exponentially with the size of the problem. No algorithm has been discovered so far to obtain the solution of them in a time of polynomial order of the problem size. A lot of computational methods have been proposed to obtain an approximate solution for such hard problems. For instance, simulated thermal annealing [1] is popular as a heuristic method.

Some of the methods for combinatorial optimization problems, including simulated thermal annealing, originate in physics. In terms of physics, the problem is encoded using by a random Ising spin Hamiltonian,

$$
\mathscr{H}_{0}=-\sum_{i} J_{i} \sigma_{i}^{z}-\sum_{i j} J_{i j} \sigma_{i}^{z} \sigma_{j}^{z}-\sum_{i j k} J_{i j k} \sigma_{i}^{z} \sigma_{j}^{z} \sigma_{k}^{z}-\cdots,
$$

where $\sigma_{i}^{z}$ is the Ising spin variable and $J_{i}, J_{i j}, J_{i j k}, \cdots$ are random coupling constants. The problem is to obtain the spin configuration of the ground state of this Hamiltonian.

Quantum annealing has been proposed originally by analogy with simulated thermal annealing [2,3]. It is also called the quantum adiabatic computation [4]. While thermal annealing introduces thermal fluctuations, quantum annealing exploits quantum fluctuations in order to achieve global optimization. It is known that thermal and quantum fluctuations give rise to similar effects in the Ising spin system. However, in spite of the resemblance, the mechanism of optimization is quite different. In quantum annealing, the solution is obtained from pursuing the time evolution of the quantum state governed by the Schrödinger equation. The optimization results from the adiabatic evolution of the quantum mechanical state [4]. Since the solution corresponding to a classical state is obtained through quantum states, quantum annealing is a so-called quantum algorithm for classical problems.

For any algorithm, it is important to reveal how powerful it is. The power of an algorithm can be determined by following properties.

(i) How the time to obtain the solution with a certain accuracy increases with the size of the problem.

(ii) How the accuracy of the solution improves with run-time.

Regarding to the property (i), Farhi et al. have reported that the time to obtain the solution of an NP-complete problem with a fixed accuracy in average increases polynomially with the size of the problem [4]. This study was done using numerical calculations for problems of small size up to about twenty spins. This result implies that the problem can be solved in a polynomial time (time of polynomial order of the problem size) in average. However another result has been reported for another NP-complete problem in contrast [5]. The exponential behavior has been observed between time and the problem size. This controversy has not been settled yet. In order to evaluate the property (ii), residual energy is appropriate. Residual energy is defined by the energy difference between an approximate solution obtained using the algorithm and the true solution. Small residual energy corresponds to high accuracy. It has been predicted for quantum annealing that residual energy decreases with run-time, $\tau$, as [6]

\footnotetext{
* E-mail: sei@stat.phys.titech.ac.jp

† E-mail: okada@k.u-tokyo.ac.jp
} 


$$
E_{\text {res }} \sim \frac{1}{(\ln \tau)^{6}}
$$

The logarithmic scaling was recently supported by the quantum Monte-Carlo simulation [7], though the evolution of state in quantum Monte-Carlo is different from the one ruled by the Schrödinger equation. In contrast, the authors of this paper have shown another scaling law of residual energy given by

$$
E_{\mathrm{res}} \sim \frac{1}{\tau^{2}}
$$

which has been supported by numerical calculations on the Schrödinger equation [8]. Since theoretical grounds of these two formulas, Eqs. (2) and (3), are different, they are not in contradiction. The latter is valid for $\tau \rightarrow \infty$, while the former is predicted for finite $\tau$ region. The behavior of residual energy in wide time region should be clarified numerically. One of the purposes of this paper is to present a feature of residual energy following the Schrödinger's real-time evolution for finite $\tau$. Note here that Eq. (3) means that the residual energy decreases much faster by means of quantum annealing in comparison with logarithmic behavior due to the thermal annealing algorithm, $E_{\text {res }} \sim 1 /(\ln \tau)^{\zeta}$, $1 \leq \zeta \leq 2$

One aspect of quantum annealing lies in possibility of implementation in classical computers. In order to carry out the algorithm, we have to calculate time evolution of a quantum state. If we do it straightforwardly, the number of bases increases exponentially and thus the simulation is limited to small sizes. In order to reveal the intrinsic power of the algorithm and also to solve problems of large size, we require a numerical method which enables to calculate real-time evolution of the quantum mechanical state for large systems. In this paper, we propose application of the density matrix renormalization group method (DMRG) for numerical implementation of the quantum annealing. DMRG has been developed in study of one-dimensional quantum systems in solid state physics. It was arranged for calculation of realtime evolution of quantum states. It may be a unique method which enables to simulate the real-time quantum dynamics in systems of large size.

We intend in this paper to explain quantum annealing and the DMRG method for combinatorial optimization problems. The next section is assigned to explanation of quantum annealing, while DMRG is introduced in Sec. 3. We also show results of simulation in latter sections. The model employed as an exercise of quantum annealing by DMRG is described in Sec. 4. The consistency of DMRG calculation is checked in Sec. 5. Finally the result of simulation is presented in Sec. 6. Section 7 is devoted to conclusion.

\section{Quantum Annealing}

We consider a general classical Hamiltonian $\mathscr{H}_{0}$ written as Eq. (1). In the quantum mechanics, the Ising spin variable, $\sigma_{i}^{z}$, is interpreted as the $z$-component of Pauli matrix and the state is represented by the state vector which defines the Hamiltonian matrix. The bases of the system are given by Ising spin configurations. The Ising spin states are given by eigenvectors of Pauli matrix $\sigma_{i}^{z}$. We denote them by $\alpha(i)$ for eigenvalue +1 and $\beta(i)$ for -1 . A basis is composed of the direct product of $\alpha(i)$ or $\beta(i)$ over all sites $i$. This basis is an eigenvector of the Hamiltonian matrix $\mathscr{H}_{0}$, since $\mathscr{H}_{0}$ is written by $\left\{\sigma_{i}^{z}\right\}$ only. The problem is to find out the ground eigenvector of $\mathscr{H}_{0}$. We introduce a tunneling Hamiltonian $\mathscr{H}_{T}$, which is responsible for quantum fluctuations in the classical system represented by $\mathscr{H}_{0}$.

$$
\mathscr{H}_{T}=-\alpha \sum_{i} \sigma_{i}^{x}
$$

where $\sigma_{i}^{x}$ is the $x$-component of the Pauli matrix with the site index $i$, and $\alpha$ is a parameter corresponding to the tunneling strength. $\sigma_{i}^{x}$ is off-diagonal with respect to $\alpha(i)$ and $\beta(i)$. Explicitly we have $\left(\alpha(i), \sigma_{i}^{x} \beta(i)\right)=(\beta(i)$, $\left.\sigma_{i}^{x} \alpha(i)\right)=1$ and $\left(\alpha(i), \sigma_{i}^{x} \alpha(i)\right)=\left(\beta(i), \sigma_{i}^{x} \beta(i)\right)=0$, where the inner product among two vectors $u$ and $v$ is defined by $(u, v) \equiv\left(u^{*}\right)^{t} v=\sum_{l} u_{l}^{*} v_{l}$. The eigenvectors of $\alpha_{i}$ are written as $\alpha(i)+\beta(i)$ and $\alpha(i)-\beta(i)$ for eigenvalues +1 and -1 respectively. The former corresponds to the state that the spin is parallel to the $x$-axis, while the latter to the anti-parallel state. The tunneling Hamiltonian given by Eq. (4) is the simplest, though there are degrees of freedom for choice of the tunneling Hamiltonian.

Using the classical and tunneling Hamiltonians, we construct the time-dependent Hamiltonian.

$$
\mathscr{H}(t)=\left(1-\frac{t}{\tau}\right) \mathscr{H}_{T}+\frac{t}{\tau} \mathscr{H}_{0},
$$

where we introduced the run-time $\tau$. The time-dependent Hamiltonian $\mathscr{H}(t)$ is defined from $t=0$ to $t=\tau$. $\mathscr{H}(t)$ is identical with the tunneling Hamiltonian at initial time, $\mathscr{H}(0)=\mathscr{H}_{T}$. On the other hand, it becomes the classical Hamiltonian at final time, $\mathscr{H}(\tau)=\mathscr{H}_{0}$.

We denote the state vector by $\Psi(t)$. As the initial condition of $\Psi(t)$, we assume that the state is at the ground state of the time-dependent Hamiltonian at $t=0$. Since $\mathscr{H}(0)=\mathscr{H}_{T}$ and all spins are aligned along $x$-axis in the ground state of $\mathcal{H}_{T}$, the initial condition is written explicitly as 


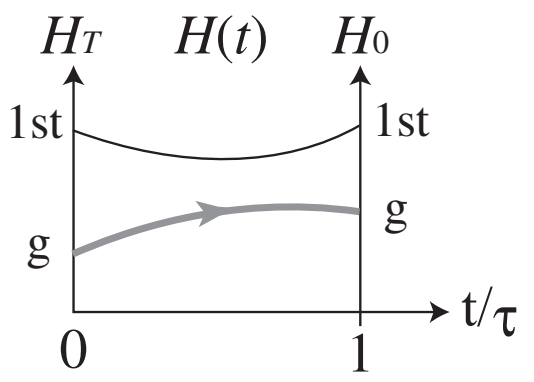

Fig. 1. A picture describing the adiabatic evolution of a quantum state. Instantaneous eigenenergies of the ground and first excited states of $\mathscr{H}(t)$ are depicted. The state evolves adiabatically from the ground state of $\mathscr{H}_{T}$ to that of $\mathscr{H}_{0}$ if the energy gap above the instantaneous ground state is finite and change of $\mathscr{H}(t)$ with $t$ is sufficiently slow.

$$
\Psi(0)=\bigotimes_{i}\left[\frac{\alpha(i)+\beta(i)}{\sqrt{2}}\right]
$$

The initial state is the quantum mechanical superposition of all possible Ising spin states. In other words, it is the linear combination with the same weight of all Ising basis vectors.

The time evolution of the state vector is governed by the Schrödinger equation.

$$
i \frac{d}{d t} \Psi(t)=\mathscr{H}(t) \Psi(t) .
$$

The final state after time evolution is read out as the approximate solution of the problem.

The mechanism of quantum annealing is described by the quantum mechanical adiabatic evolution of the state. By assumption, the state is initially at the ground eigenvector of the time-dependent Hamiltonian. If finite energy gap separates the instantaneous ground state and first excited state of the time-dependent Hamiltonian, the state evolves adiabatically and arrives at the ground state of the classical Hamiltonian when the time dependence of the Hamiltonian is sufficiently moderate. We display a conceptual picture of quantum annealing in Fig. 1. We recall that the timedependent Hamiltonian, Eq. (5), is linear in time and its gradient by time is proportional to $1 / \tau$. Hence the timedependent Hamiltonian changes slower with time for longer $\tau$. The criterion of adiabatic evolution is given by $\tau \gg \tau_{C}$, where $\tau_{C}$ is a characteristic time defined by $\tau_{C}=\varepsilon^{\prime} / \varepsilon_{\min }^{2}$ with $\varepsilon_{\min }$, the minimum energy gap above the instantaneous ground state, and $\varepsilon^{\prime}$, maximum of the matrix element of $\mathcal{H}_{T}-\mathscr{H}_{0}$ among the instantaneous ground state and the first excited state. The convergence of the final state to the ground state of $\mathscr{H}_{0}$ for $\tau \rightarrow \infty$ is guaranteed by the adiabatic theorem in quantum mechanics as far as $\varepsilon_{\min }$ is finite. If the ground state of $\mathcal{H}_{0}$ is not degenerated, $\varepsilon_{\min }$ does not vanish since level crossing between the instantaneous ground state and first excited state is avoided due to quantum fluctuations.

\section{Density Matrix Renormalization Group Method}

In order to implement quantum annealing, we have to calculate time evolution of the state vector. Because of exponential increase in the number of bases, the direct solution of the Schrödinger's differential equation, Eq. (6), is unavailable except for small systems. The density matrix renormalization group (DMRG) method is probably the unique method which enables us to calculate the time evolution of state vector for quantum spin systems of large size. DMRG has been developed originally to obtain low energy states of one-dimensional quantum systems, and arranged for calculation of time evolution of a quantum state recently [9]. We apply the method of DMRG to quantum annealing.

We consider an evolution according to the Schrödinger equation during a short time from $t$ to $t+\Delta t$. The evolved state is written approximately using time-evolution operator $e^{-i \mathscr{H}(t) \Delta t}$ as

$$
\Psi(t+\Delta t) \cong e^{-i \mathscr{H}(t) \Delta t} \Psi(t)
$$

This formula holds exactly, if the Hamiltonian $\mathscr{H}(t)$ is independent of time during $[t, t+\Delta t]$. Hence this approximation indicates discretization of $\mathscr{H}(t)$, that is defined by

$$
\overline{\mathscr{H}}(t)=\mathscr{H}(l \Delta t) \quad(l \Delta t \leq t<(l+1) \Delta t) .
$$

The discrete Hamiltonian represents stepwise change of the parameter in system such as the magnetic field. We note that it is physically possible to change the magnetic field discontinuously. Although such a discontinuous change of the Hamiltonian is unfavorable for the adiabatic motion of state, we expect that the adiabaticity is preserved for sufficiently short time width $\Delta t$. We thus define quantum annealing by this discrete process hereafter.

The final state after evolution from $t=0$ to $t=\tau$ is given by 


$$
\Psi(\tau)=e^{-i \mathscr{H}\left(\left(N_{t}-1\right) \Delta t\right) \Delta t} \cdots e^{-i \mathscr{H}(\Delta t) \Delta t} e^{-i \mathscr{H}(0) \Delta t} \Psi(0),
$$

where the Trotter number $N_{t}$ satisfies $N_{t} \Delta t=\tau$. The time-dependent Hamiltonian is divided with respect to the interaction.

$$
\mathscr{H}(t)=\mathscr{H}_{1}(t)+\mathscr{H}_{2}(t)+\cdots,
$$

where each term in the right hand side contains one multi-spin interaction term and single-spin terms. The timeevolution operator is decomposed symmetrically into a product of time-evolution operators of $\mathcal{H}_{j}(t)$.

$$
e^{-i \mathscr{H}(t) \Delta t} \cong e^{-i \mathscr{H}_{1}(t) \frac{\Delta t}{2}} \cdots e^{-i \mathscr{H}_{M-1}(t) \frac{\Delta t}{2}} e^{-i \mathscr{H}_{M}(t) \Delta t} e^{-i \mathscr{H}_{M-1}(t) \frac{\Delta t}{2}} \cdots e^{-i \mathscr{H}_{1}(t) \frac{\Delta t}{2}}
$$

where $M$ is the number of interaction terms in $\mathscr{H}_{0}$.

Now we consider the one-dimensional lattice. We assume for simplicity that $\mathscr{H}_{0}$ contains only terms of pair interaction between nearest-neighbors and single-spin terms. We take a look at $j$-th and $(j+1)$-th sites. In DMRG, onedimensional lattice is divided into four blocks, namely left block, $j$-th site, $(j+1)$-th site, and right block. We denote the bases of these blocks by $\psi_{a}^{L}, \psi_{k}^{j}, \psi_{l}^{j+1}$, and $\psi_{b}^{R}$, respectively. The state vector is represented as

$$
\Phi=\sum_{a, b, k, l} \phi_{a, b, k, l} \psi_{a}^{L} \psi_{k}^{j} \psi_{l}^{j+1} \psi_{b}^{R}
$$

We do an operation of $e^{-i \mathscr{H}_{j}(t) \frac{\Delta t}{2}}$, supposing that $\mathscr{H}_{j}(t)$ contains the spin operators of site $j$ and $j+1$. Denoting the matrix element of $e^{-i \mathscr{H}_{j}(t) \frac{\Delta t}{2}}$ by $\left[e^{-i \mathscr{H}_{j}(t) \frac{\Delta t}{2}}\right]_{k l ; k^{\prime} l^{\prime}}$, we obtain

$$
e^{-i \mathscr{H}_{j}(t) \Delta t} \Phi=\sum_{a, b, k, l} \tilde{\phi}_{a, b, k, l} \psi_{a}^{L} \psi_{k}^{j} \psi_{l}^{j+1} \psi_{b}^{R},
$$

where

$$
\tilde{\phi}_{a, b, k, l}=\sum_{k^{\prime}, l^{\prime}}\left[e^{-i \mathscr{H}_{j}(t) \frac{\Delta t}{2}}\right]_{k l ; k^{\prime} l^{\prime}} \phi_{a, b, k^{\prime}, l^{\prime}} .
$$

We define the density matrix in terms of $\tilde{\phi}_{a, b, k, l}$ as

$$
\rho_{a k ; a^{\prime} k^{\prime}}=\sum_{b, l} \tilde{\phi}_{a, b, k, l} \tilde{\phi}_{a^{\prime}, b, k^{\prime}, l}^{*}
$$

The density matrix is diagonalized using a unitary matrix $u_{c, a k}$.

$$
\rho_{a k ; a^{\prime} k^{\prime}}=\sum_{c} u_{c, a k} d_{c} u_{c, a^{\prime} k^{\prime}}^{*}
$$

where $d_{c}$ is the eigenvalue of the density matrix. We introduce a new basis $\psi_{a}$ by the eigenstate of the density matrix. $\psi_{a}$ relates with $\psi_{a^{\prime}}^{L} \psi_{k}^{j}$ through the matrix $u_{a, a^{\prime} k}$.

$$
\psi_{a}=\sum_{a^{\prime}, k} u_{a, a^{\prime} k} \psi_{a^{\prime}}^{L} \psi_{k}^{j}
$$

We employ $\psi_{a}^{L^{\prime}} \equiv \psi_{a}$ as the basis of the new left block composed of the old left block and the site $j$. The right block is divided into one spin at the left side and new right block. We denote the bases of these new blocks by $\psi_{l}^{j+2}$ and $\psi_{b}^{R^{\prime}}$. The basis of old right block is written using new bases as

$$
\psi_{b}^{R}=\sum_{l, b^{\prime}} v_{b, l b^{\prime}} \psi_{l}^{j+2} \psi_{b^{\prime}}^{R^{\prime}}
$$

where $v_{b, l b^{\prime}}$ is a unitary matrix which diagonalizes the density matrix with respect to the site $(j+2)$ and the right block $R^{\prime} . v_{b, l b^{\prime}}$ is supposed to have been obtained in advance. We remark that the number of spins in $\psi_{b}^{R^{\prime}}$ in the right hand side is less than that in $\psi_{b}^{R}$ in the left hand side by one. Using the new bases, $\psi_{a}^{L^{\prime}}, \psi_{k}^{j+1}, \psi_{l}^{j+2}$, and $\psi_{b}^{R^{\prime}}$, we express the state vector as

$$
\begin{gathered}
\sum_{a, b, k, l} \tilde{\phi}_{a, b, k, l} \psi_{a}^{L} \psi_{k}^{j} \psi_{l}^{j+1} \psi_{b}^{R}=\sum_{a, b, k, l} \phi_{a, b, k, l}^{\prime} \psi_{a}^{L^{\prime}} \psi_{k}^{j+1} \psi_{l}^{j+2} \psi_{b}^{R^{\prime}}, \\
\phi_{a, b, k, l}^{\prime}=\sum_{a^{\prime}, b^{\prime}, k^{\prime}} \tilde{\phi}_{a^{\prime}, b^{\prime}, k^{\prime}, k} u_{a, a^{\prime} k^{\prime}}^{*} v_{b^{\prime}, l b}
\end{gathered}
$$

The norm of the state vector is written in terms of the eigenvalue of the density matrix.

$$
\sum_{a, b, k, l} \tilde{\phi}_{a, b, k, l} \tilde{\phi}_{a, b, k, l}^{*}=\sum_{a} d_{a}
$$

The eigenvalue $d_{a}$ is a positive number. If $d_{a}$ is negligibly small, the norm of the state vector is not affected by the 
absence of the state $\psi_{a}^{L^{\prime}}$. The procedure of density matrix renormalization is to remove bases with small eigenvalue so as to preserve the norm. As far as the number of dominant eigenvalues is kept small, it is allowed to keep the number of bases to a certain tractable number $m$. Thus we transform the basis and move to next operation of $e^{-i \mathcal{H}_{j+1}(t) \Delta t}$. The operation of one time-evolution operator, Eq. (9), is accomplished by the right-ward sweep followed by the left-ward sweep.

\section{Model}

We take up the ground state search of random field Ising model in one dimension as an exercise for quantum annealing using DMRG. An efficient algorithm is present for the ground state problem of random field Ising model [10]. One can obtain the ground state by means of this algorithm in a time which scales by a polynomial of the number of spins, $N$. We note that the aim of the present study is not to find the solution of given combinatorial optimization problem but to carry out quantum annealing with DMRG and discuss power of the quantum annealing. To this end, it is significant to investigate essential features of the method for an well-known exercise, even though it is not a hard instance.

The Hamiltonian of the random field Ising model is written by

$$
\mathscr{H}_{0}=-J \sum_{i=1}^{N-1} \sigma_{i}^{z} \sigma_{i+1}^{z}-\sum_{i=1}^{N} h_{i} \sigma_{i}^{z},
$$

where $\sigma_{i}^{z}$ indicates the Ising variable in the classical mechanics or the $z$-component of the Pauli matrix in the quantum mechanics as was mentioned in Sec. 2. The ferromagnetic interaction works only between nearest-neighbor spin pairs, and is uniform. The on-site magnetic field $h_{i}$, which is responsible for the randomness of the system, takes the value +1 or -1 with the same probability. The problem is to find the Ising-spin configuration of the ground state of this Hamiltonian. If $J$ is sufficiently large, the ground state of a finite-size system is ferromagnetic $\left(\frac{1}{N} \sum_{i}\left|\sigma_{i}\right|=1\right)$. On the other hand, all spins are independent and parallel to the magnetic field in the ground state of the absence of $J$. For a certain intermediate value of $J$, the ground state is non-trivial.

In advance of simulation of quantum annealing, we first examine the ground state for a instance of random magnetic field using an efficient algorithm described in Ref. 10, and get rid of the instance which gives rise to degenerate ground states. This is because the instance with degenerate ground states could be easier than that with unique solution and thus give rise to large fluctuation in difficulty of the problem.

\section{Consistency and Choice of Parameters}

In order to appeal the consistency of DMRG calculation, we first show the result on nine spin system in Fig. 2. We can solve the Schrödinger equation, Eq. (6), directly for nine spin system by means of the Runge-Kutta method. Results of DMRG and Runge-Kutta methods are shown in the figure. There exist two parameters in DMRG calculation, i.e., the time-slice width $\Delta t$ and the number of bases kept in density matrix renormalization $m$. The result of DMRG in Fig. 2 is with $\Delta t=0.1$ and $m=16$. The overlap of abscissa is defined by the absolute square of inner product between the state vector at the final time and the true ground state. It is expected that the overlap increases with the run-time $\tau$ and approaches to 1 for $\tau \rightarrow \infty$. We see in Fig. 2 that the overlap obtained by DMRG shown by square symbols is in good agreement with that obtained by Runge-Kutta method shown by the curve.

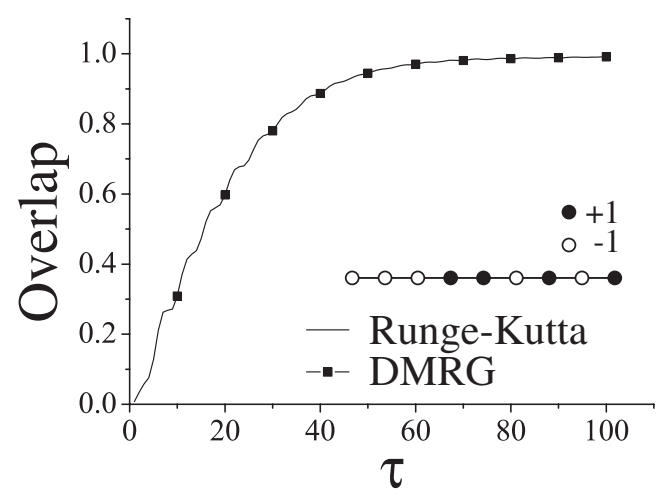

Fig. 2. Overlap, $\left|\left(\Psi(\tau), \Psi_{g}\right)\right|^{2}$, between the state vector at the final time $\Psi(\tau)$ and the true ground state $\Psi_{g}$ for one-dimensional random field Ising model with nine spins. The ferromagnetic coupling constant $J$ between nearest neighbor spins is set at 0.6. The configuration of random field is depicted in the figure. The ordinate is the run-time $\tau$. The curve in the figure is the result obtained by the Runge-Kutta method. The square symbols at $\tau=10,20, \ldots$, and 100 are obtained by the DMRG method with $\Delta t=0.1$ and $m=16$. The result of DMRG is in good agreement with the direct calculation by Runge-Kutta method. 


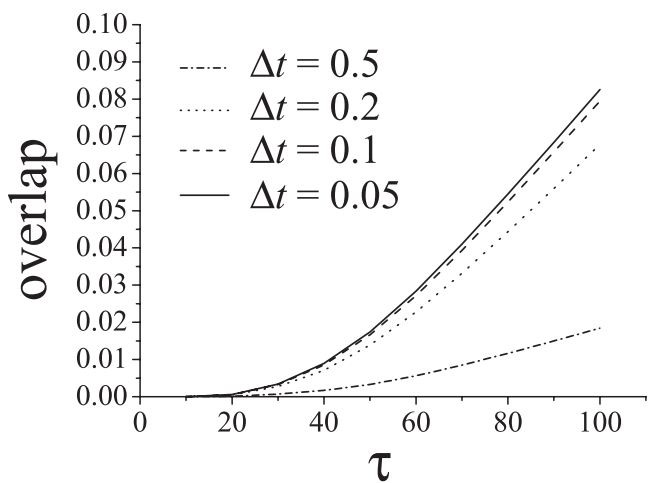

Fig. 3. Overlaps obtained by DMRG with $\Delta t=0.05,0.1,0.2$, and 0.5 and $m=16$ for $N=100$ spins. We gave $J=1.6$ for the coupling constant for the Ising model. We omit an exhibition of the configuration of random field. The difference of results with $\Delta t=0.05$ and 0.1 is small compared to that of $\Delta t=0.1$ and 0.2 . We infer that the time evolution of $\Delta=0.1$ mimics the continuous-time evolution. We have also performed the same calculation for $m=32$. Results are not shown, but they are indistinguishable even if they are present. Hence $m=16$ is sufficient to produce precise results.

Two parameters, $\Delta t$ and $m$, may influence the accuracy of DMRG calculation. As has been mentioned, $\Delta t$ determines discontinuity of the Hamiltonian. The continuous-time evolution is recovered for $\Delta t \rightarrow 0$. Figure 3 shows overlaps of several $\Delta t$ 's and $m=16$ for $N=100$ spins obtained by DMRG. The overlap increases monotonically with decreasing $\Delta t$. To perform the continuous-time evolution, one should set $\Delta t$ as small as possible. It is found in the figure that the difference between results of $\Delta t=0.05$ and 0.1 is small compared to that between $\Delta t=0.1$ and 0.2 . We infer that $\Delta t=0.1$ is fairly small to perform time evolution which mimics the continuous-time evolution. Hence we settle $\Delta t=0.1$ in practice in the following DMRG calculation. For given $\Delta t$, it is not obvious how large value should one give for $m$. We have also performed calculations same as those shown in Fig. 3 but with $m=32$. The difference of results of $m=32$ and $m=16$ are invisible even if they are drawn in the same figure. Hence we expect that $m=16$ is sufficient to produce precise results.

\section{Results}

We calculated residual energy by DMRG for $N=350$ spins. Residual energy after quantum annealing is defined by the energy expectation value of the state vector at final time measured from the true ground state,

$$
E_{\text {res }}=\left(\Psi(\tau), \mathscr{H}_{0} \Psi(\tau)\right)-E_{g},
$$

where $E_{g}$ indicates the true ground energy. The ferromagnetic coupling constant $J$ was settled at $J=1.6$ in the simulation. For the random field, eighty instances that has the unique ground state were generated. We take the average over the eighty instances. DMRG parameters were settled at $\Delta t=0.1$ and $m=16$.

Figure 4 shows run-time dependence of averaged residual energy. Square symbols connected by line are the results by the DMRG calculation. Remark that the residual energy of abscissa is in logarithmic scale and the ordinate is $\log _{10} \tau$

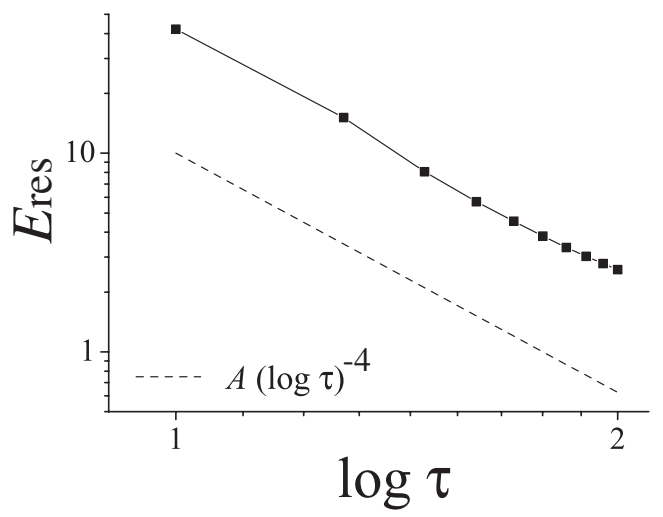

Fig. 4. Run-time dependence of the averaged residual energy obtained by DMRG (squares) for $N=350$ spins. The coupling constant $J$ is settled at $J=1.6$. The average was taken over eighty instances of random field which gives rise to a unique ground state. A line proportional to $\left(\log _{10} \tau\right)^{-4}$ is plotted for reference. It is found that the residual energy decays almost in proportion to $(\ln \tau)^{-4}$. 
in logarithmic scale. Clearly residual energy decreases monotonically with run-time. It is likely that $\log _{10} E_{\text {res }}$ is linear with respect to $\log _{10} \tau$. We show the line of $A\left(\log _{10} \tau\right)^{-4}$ with a constant $A$ in the figure for comparison. From this we find that the gradient is almost -4 , namely we obtain

$$
E_{\text {res }} \sim \frac{1}{(\ln \tau)^{4}}
$$

The logarithmic law of the residual energy has been predicted by Santoro et al. analytically for two-dimensional spin-glass model [6], and reported by Sarjala et al. numerically for random field Ising model [7]. The theory in the former is based on the Schrödinger's real-time dynamics and derives the exponent $\zeta=6$. The latter relies on the Markovian dynamics of the quantum Monte-Carlo and exponent is smaller than 6. Our result is from the real-time dynamics. The exponent is in conflict with the analytical prediction but rather comparable to the result of quantum Monte-Carlo.

When the ferromagnetic coupling constant is at $J=1.6$, the ground state of the one-dimensional model consists of ferromagnetic domains. The presence of ferromagnetic domains implies occurrence of Landau-Zener level-cross avoiding during the annealing process. The theory of Ref. 6 is grounded on the Landau-Zener theory and hence should cover the problem we are studying. In order to resolve the discrepancy between our result and this theory, following two scenarios are considered. The first is that $\zeta=4$ is a universal value and the theory should be corrected. The second is that $\zeta$ decreases from $\zeta_{\max }=6$ with increasing $J$ from $J=0$. The study by quantum Monte-Carlo simulation suggests the second scenario [7]. To give a definite answer, we need to carry out DMRG calculation on other $J$ 's.

We remark that the logarithmic law presented here is not in conflict with the power law which was reported in Ref. 8. The power law is the property which emerges in long limit region of run-time $\tau$. The run-time presented in Fig. 4 is much shorter. The power law should be observed for long run-time limit even in our simulation.

\section{Conclusion}

We applied the density matrix renormalization group (DMRG) for simulation of quantum annealing. DMRG is a promising method of simulation for one-dimensional quantum spin systems. We confirmed consistency of DMRG calculation for the problem of ground state search in one-dimensional random-field Ising model. Using this method, we investigated residual energy after quantum annealing for one-dimensional random-field Ising model. The result for problems with 350 spins shows that residual energy decays with run-time as $1 /(\ln \tau)^{-4}$. This is the first observation that follows the real-time evolution for the problem of hundreds of spins. The logarithmic law of residual energy is in consistent with the earlier prediction, but the exponent is inconsistent.

DMRG enables us to carry out quantum annealing by real-time evolution for one-dimensional problems. We expect that this method leads to further understanding of quantum annealing.

\section{Acknowledgments}

This work was partially supported by a Grant-in-Aid for Scientific Research on Priority Areas No. 18020007 and 18079003, and a Grant-in-Aid for Scientific Research (C) No. 16500093.

\section{REFERENCES}

[1] Kirkpatrick, S., Gelett, C. D., and Vecchi, M. P., Science, 220: 671 (1983).

[2] Finnila, A. B., Gomez, M. A., Sebenik, C., Stenson, C., and Doll, J. D., Chem. Phys. Lett., 219: 343 (1994).

[3] Kadowaki, T., and Nishimori, H., Phys. Rev. E, 58: 5355 (1998).

[4] Farhi, E., Goldstone, J., Gutmann, S., Lapan, J., Lundgren, A., and Preda, D., Science, 292: 472 (2001).

[5] Smelyanskiy, V. N., Toussaint, U. V., and Timucin, D. A., quant-ph/0112143.

[6] Santoro, G. E., Martoňák, R., Tosatti, E., and Car, R., Science, 295: 2427 (2002).

[7] Sarjala, M., Petäjä, V., and Alava, M., J. Stat. Mech., 1008 (2006).

[8] Suzuki, S., and Okada, M., J. Phys. Soc. Jpn., 74: 1649 (2005).

[9] White, S. R., and Feiguin, A. E., Phys. Rev. Lett., 93: 076401 (2004).

[10] Alava, M., Duxbury, P., Moukarzel, C., and Rieger, H., in Phase Transitions and Ciritical Phenomena vol. 18, ed. C. Domb and J. L. Lebowitz (2001, San Diego, CA: Academic). 